\title{
UNBOUNDED DERIVED CATEGORIES AND THE FINITISTIC DIMENSION CONJECTURE
}

\author{
JEREMY RICKARD
}

\begin{abstract}
We consider the question of whether the injective modules generate the unbounded derived category of a ring as a triangulated category with arbitrary coproducts. We give an example of a nonNoetherian commutative ring where they don't, but prove that they do for any Noetherian commutative ring. For non-commutative finite dimensional algebras the question is open, and we prove that if injectives generate for such an algebra, then the finitistic dimension conjecture holds for that algebra.
\end{abstract}

\section{INTRODUCTION}

In the representation theory of finite dimensional algebras, a circle of interconnected questions and conjectures collectively known as the "homological conjectures" have inspired a lot of work over the last few decades. In the 1980s derived categories were introduced into representation theory, and it was natural to try to interpret these homological conjectures in terms of derived categories. Happel [Hap91, [Hap93 related many of these conjectures to bounded derived categories and in Hap91] gives a good overview of the conjectures.

More recently, it has increasingly become clear that it is often more natural and convenient to work with the unbounded derived category of complexes of arbitrary modules, since this has better properties, especially having arbitrary coproducts.

In a talk in Nordfjordeid in 2001 [Kel01, Keller considered the question of whether the unbounded derived category is generated, as a triangulated category with infinite coproducts, by the injective modules (if this is the case, then we say that "injectives generate"), and pointed out that if this were the case then some of the homological conjectures for finite dimensional algebras would follow: in particular, the Nunke condition that for any finitely generated nonzero module $X$ for a finite dimensional algebra $A, \operatorname{Ext}_{A}^{i}(D A, X) \neq 0$ for some $i \geq 0$, where $D A$ is the dual of the regular module, and a fortiori the generalized Nakayama conjecture that for every simple $A$-module $S$, $\operatorname{Ext}_{A}^{i}(D A, S) \neq 0$ for some $i \geq 0$. He asked whether there was any connection to the finitistic dimension conjecture, which is known to imply many of the other homological conjectures, including the Nunke condition and generalized Nakayama conjecture.

Date: April 27, 2018.

Key words and phrases. Finite dimensional algebras; derived categories; finitistic dimension conjecture. 
In this paper we answer this question in Theorem 4.3 by proving that if injectives generate for a finite dimensional algebra $A$ then the finitistic dimension conjecture holds for $A$. At present I know no examples of finite dimensional algebras for which injectives do not generate.

We first, in Section 3, consider the question of whether injective modules generate the derived category for more general rings. Our main results are a proof that they always do for commutative Noetherian rings, and an example of a commutative non-Noetherian ring where they don't.

In Section 5 we briefly consider the dual condition of projective modules generating the derived category as a triangulated category with products. It is not clear that the two conditions are equivalent, but for finite dimensional algebras we prove that "injectives generate" implies the dual condition.

In Section 6 6 we work though an example of a finite dimensional algebra for which injectives generate to illustrate some of the more elementary techniques that can be used.

Finally we develop some techniques for proving, for particular finite dimensional algebras, that injective modules generate the derived category, and use these to prove that injectives generate for some classes of algebras where the finitistic dimension conjecture is known to hold. In some of these, the finitistic dimension conjecture is obvious, but it is less obvious that injectives generate, and there are also several classes of algebras for which the finitistic dimension conjecture is known to hold but for which I don't know whether injectives generate.

\section{ACKNOWLEDGEMENTS}

I would like to thank Birge Huisgen-Zimmermann, Bernhard Keller, Henning Krause and Greg Stevenson for conversations and email exchanges related to the contents of this paper.

\section{Preliminaries}

In this section we shall fix some notation and discuss some generalities on localizing subcategories.

Let $R$ be a ring, not necessarily commutative. We shall be considering $R$ modules and complexes of $R$-modules, and our standard conventions will be that by an $R$-module we mean a right $R$-module, and by a complex we mean a cochain complex, unless we specify otherwise. We shall use the following notation for various categories related to $R$.

- Mod- $R$ is the category of (right) $R$-modules.

- $\mathcal{K}(R)=\mathcal{K}(\operatorname{Mod}-R)$ is the homotopy category of (cochain) complexes of $R$-modules.

- $\mathcal{D}(R)=\mathcal{D}(\operatorname{Mod}-R)$ is the derived category of (cochain) complexes of $R$-modules.

We impose no finiteness conditions on the modules in Mod- $R$ and no boundedness conditions on the complexes in $\mathcal{K}(R)$ and $\mathcal{D}(R)$.

We shall regard Mod- $R$ as a full subcategory of both $\mathcal{K}(R)$ and $\mathcal{D}(R)$ in the usual way, identifying a module $M$ with the complex which has $M$ in degree zero and zero in all other degrees. 
The derived category $\mathcal{D}(R)$ is a triangulated category with arbitrary (small) coproducts, so it makes sense to consider the localizing subcategory generated by a particular class $\mathcal{S}$ of objects (i.e., the smallest triangulated subcategory of $\mathcal{D}(R)$ that contains $\mathcal{S}$ and is closed under coproducts) and to ask whether this is the whole of $\mathcal{D}(R)$. We shall denote by $\langle\mathcal{S}\rangle$ the localizing subcategory generated by $\mathcal{S}$, and if this is the whole of $\mathcal{D}(R)$ then we shall say that " $\mathcal{S}$ generates $\mathcal{D}(R)$ ". There are other notions of generation for triangulated categories, but this is the only one that we shall consider here, except briefly in Section 5 .

We shall start by considering some easy concrete properties of localizing subcategories, none of which are original, which will be useful when we come to look at examples.

Proposition 2.1. Let $\mathcal{C}$ be a localizing subcategory of $\mathcal{D}(R)$.

a) If $0 \rightarrow X \rightarrow Y \rightarrow Z \rightarrow 0$ is a short exact sequence of complexes, and two of the three objects $X, Y$ and $Z$ are in $\mathcal{C}$, then so is the third.

b) If a complex $X$ is in $\mathcal{C}$ then so is the shifted complex $X[t]$ for every $t \in \mathbb{Z}$.

c) If $X$ and $Y$ are quasi-isomorphic complexes and $X$ is in $\mathcal{C}$, then so is $Y$.

d) If $\left\{X_{i} \mid i \in I\right\}$ is a set of objects of $\mathcal{C}$, then $\bigoplus_{i \in I} X_{i}$ is in $\mathcal{C}$.

e) If $X \oplus Y$ is in $\mathcal{C}$ then so are $X$ and $Y$.

f) If $X=X^{*}$ is a bounded complex, where the module $X^{i}$ is in $\mathcal{C}$ for every $i$, then $X$ is in $\mathcal{C}$.

g) If $X_{0} \stackrel{\alpha_{0}}{\longrightarrow} X_{1} \stackrel{\alpha_{1}}{\longrightarrow} X_{2} \stackrel{\alpha_{2}}{\longrightarrow} \ldots$ is a sequence of cochain maps between complexes, with $X_{i}$ in $\mathcal{C}$ for all $i$, then $\lim _{i} X_{i}$ is in $\mathcal{C}$.

h) If $X:=X^{*}$ is a bounded above complex, where the module $X^{i}$ is in $\mathcal{C}$ for every $i$, then $X$ is in $\mathcal{C}$.

Proof. (a), (b) and (c) are just restatements of the definition of a triangulated subcategory, since a short exact sequence of complexes becomes a distinguished triangle in the derived category, and (d) is the definition of what it means for a triangulated subcategory to be localizing.

There is a short exact sequence

$$
0 \rightarrow X \rightarrow(X \oplus Y \oplus X \oplus \ldots) \rightarrow(Y \oplus X \oplus Y \oplus \ldots) \rightarrow 0
$$

where the last two terms are both coproducts of countably many copies of $X \oplus Y$, so (e) follows from (a) and (d). This trick is a form of the "Eilenberg swindle".

Using (a) and (b), an easy induction on the length of a bounded complex $X^{*}$ proves (f), since if $n$ is maximal with $X^{n} \neq 0$, then there is an exact sequence of complexes

$$
0 \rightarrow X^{n}[-n] \rightarrow X^{*} \rightarrow \sigma^{<n} X^{*} \rightarrow 0,
$$

where $\sigma^{<n} X^{*}=\cdots \rightarrow X^{n-2} \rightarrow X^{n-1} \rightarrow 0 \rightarrow \ldots$ is the "brutal" truncation of $X^{*}$.

For $(\mathrm{g})$, there is a short exact sequence

$$
0 \rightarrow \bigoplus_{i=0}^{\infty} X_{i} \rightarrow \bigoplus_{i=0}^{\infty} X_{i} \rightarrow \lim _{\longrightarrow} X_{i} \rightarrow 0
$$


where the first map is $\alpha_{n}-\mathrm{id} X_{n}$ on $X_{n}$, and so the claim follows from (a) and (d). Note that this is a special case of the fact that a localizing subcategory is closed under homotopy colimits (see, for example, BN93]) of sequences of maps.

A bounded above complex $X^{*}$ is the direct limit of its brutal truncations $\sigma^{\geq n} X^{*}=\cdots \rightarrow 0 \rightarrow X^{n} \rightarrow X^{n+1} \rightarrow \ldots$, which are bounded, and so (h) follows from (f) and (g).

It is important to note that the proof of $(\mathrm{h})$ would not work (and in fact the statement would be false in general) if we replaced "bounded above" by "bounded below", since a bounded below complex is the inverse limit, rather than direct limit, of its bounded brutal truncations. It would be true if we also replaced "localizing subcategory" by "colocalizing subcategory" (i.e., triangulated subcategory closed under products).

It is well-known that $\mathcal{D}(R)$ is generated (as a triangulated category with coproducts) by the projective modules. There are more sophisticated, and arguably better, ways of proving this, but we'll give a rather concrete argument, since some of the ideas will be relevant later on.

Proposition 2.2. The projective $R$-modules generate $\mathcal{D}(R)$.

Proof. Let $X^{*}$ be a complex of $R$-modules. It is the direct limit of its "good" truncations

$$
\tau^{\leq n} X^{*}=\cdots \longrightarrow X^{n-2} \stackrel{d^{n-2}}{\longrightarrow} X^{n-1} \stackrel{d^{n-1}}{\longrightarrow} \operatorname{ker} d^{n} \longrightarrow 0 \longrightarrow \ldots,
$$

which are bounded above, so by $(\mathrm{g})$ and $(\mathrm{h})$ of Proposition 2.1 it suffices to prove that every $R$-module is in the localizing subcategory generated by the projectives.

But an $R$-module $M$ is quasi-isomorphic to any projective resolution of $M$, and is therefore in the localizing subcategory generated by the projectives by (c) and (h) of Proposition 2.1.

Since every projective module is a direct summand of a direct sum of copies of the regular right $R$-module $R_{R}$, this shows that the single object $R_{R}$ generates $\mathcal{D}(R)$.

\section{The LOCALIZING SUBCATEGORY GENERATED BY INJECTIVES}

A similar proof to that of Proposition 2.2 shows that the injective $R$ modules generate $\mathcal{D}(R)$ as a triangulated category with products (i.e., the colocalizing subcategory generated by the injectives is the whole of $\mathcal{D}(R)$ ), but we shall consider the localizing subcategory generated by injectives. We'll denote by Inj- $R$ the category of injective $R$-modules, so this localizing subcategory will be denoted by $\langle\operatorname{Inj}-R\rangle$.

This paper will explore the following condition that a ring might satisfy.

Definition 3.1. If $\langle\operatorname{Inj}-R\rangle=\mathcal{D}(R)$ then we say that injectives generate for $R$.

In later sections we shall mainly consider the case of finite dimensional algebras, but in this section we shall prove a few results for arbitrary rings and give an example of a ring for which injectives do not generate. 
First, there is one class of rings, including those of finite global dimension, for which it is very easy to see that injectives generate.

Theorem 3.2. If the regular right module $R_{R}$ has finite injective dimension, then injectives generate for $R$. In particular, injectives generate for any ring with finite global dimension.

Proof. The module $R_{R}$ is quasi-isomorphic to an injective resolution, which if $R$ has finite injective dimension can be taken to be a bounded complex of injectives, which is in $\langle\operatorname{Inj}-R\rangle$ by Proposition 2.1. Since $R_{R}$ generates $\mathcal{D}(R)$ by Proposition 2.2, injectives generate.

For commutative Noetherian rings there is a classification of localizing subcategories of $\mathcal{D}(R)$ that makes it easy to see that injectives generate.

Theorem 3.3. If $R$ is a commutative Noetherian ring, then injectives generate for $R$.

Proof. Let $R$ be a commutative Noetherian ring. By a famous theorem of Hopkins Hop87 and Neeman [Nee92, there is an inclusion preserving bijection between the collection of sets of prime ideals and the collection of localizing subcategories of $\mathcal{D}(R)$, and by Lemma 2.9 of Neeman Nee92, for any prime ideal $\mathfrak{p}$, the injective hull $I(R / \mathfrak{p})$ of $R / \mathfrak{p}$ is in the localizing subcategory corresponding to $\{\mathfrak{p}\}$, which is a minimal nonzero localizing subcategory, and therefore generated by $I(R / \mathfrak{p})$. Hence the only localizing subcategory that contains all injectives is the one corresponding to $\operatorname{Spec}(R)$, the set of all prime ideals, which is $\mathcal{D}(R)$.

Next we show that if $R$ is derived equivalent to a ring for which injectives generate, then injectives generate for $R$. This follows from the fact that we can characterize the objects of the derived category isomorphic to bounded complexes of injectives.

Theorem 3.4. Let $R$ and $S$ be derived equivalent rings; i.e., $\mathcal{D}(R)$ and $\mathcal{D}(S)$ are equivalent as triangulated categories. If injectives generate for $S$ then injectives generate for $R$.

Proof. It is well-known that the compact objects of the derived category are the perfect complexes: those isomorphic to bounded complexes of finitely generated projectives.

The objects with cohomology bounded in degree are those objects $B$ such that, for each perfect complex $C, \operatorname{Hom}(C, B[t])=0$ for all but finitely many $t \in \mathbb{Z}$.

The objects isomorphic to bounded complexes of injectives (i.e., those with bounded injective resolutions) are those objects $X$ such that, for every object $B$ with bounded cohomology, $\operatorname{Hom}(B, X[t])=0$ for all but finitely many $t \in \mathbb{Z}$.

Thus an equivalence of triangulated categories between $\mathcal{D}(R)$ and $\mathcal{D}(S)$ restricts to an equivalence between the subcategories of objects isomorphic to bounded complexes of injectives. By Proposition 2.1 this subcategory generates the same localizing subcategory as the injectives, so if injectives generate for one of the two rings $R$ and $S$ then they generate for the other. 
We end this section by giving an example of a non-Noetherian commutative ring for which injectives don't generate.

Let $k$ be a field. If $V$ is a $k$-vector space, $D V$ will denote the dual space $\operatorname{Hom}_{k}(V, k)$.

Theorem 3.5. Let $R=k\left[x_{0}, x_{1}, \ldots\right]$, the ring of polynomials over $k$ in countably many variables. Injectives do not generate for $R$.

Proof. We denote by $S$ the one-dimensional $R$-module $R /\left(x_{0}, x_{1}, \ldots\right)$, and we shall show that $S$ is not in $\langle\operatorname{Inj}-R\rangle$.

It suffices to prove that $\operatorname{Hom}(X, S)=0$ for every object $X$ of $\langle\operatorname{Inj}-R\rangle$, since clearly $\operatorname{Hom}(S, S) \neq 0$.

Since the class of objects $X$ such that $\operatorname{Hom}(X, S[t])=0$ for all $t \in \mathbb{Z}$ is a localizing subcategory of $\mathcal{D}(R)$, it suffices to prove that, for every injective module $I$ and every $t \geq 0$, $\operatorname{Ext}_{R}^{t}(I, S)=\operatorname{Hom}(I, S[t])=0$ (where $\operatorname{Ext}^{0}$ denotes Hom).

Since $D R$ is an injective cogenerator of the module category, every injective $R$-module is a direct summand of some product $D R^{J}$ of copies of $D R$, so it suffices to prove that $\operatorname{Ext}_{R}^{t}\left(D R^{J}, S\right)=0$ for every set $J$ and every $t \geq 0$.

Let $C_{i}$ be the chain complex $\ldots \rightarrow 0 \rightarrow R \stackrel{x_{i}}{\rightarrow} R \rightarrow 0 \rightarrow \ldots$ with the second non-zero term in degree zero, let $X_{n}=\bigotimes_{i<n} C_{i}$, so there are natural inclusions $X_{1} \rightarrow X_{2} \rightarrow \ldots$, and let $X_{\infty}=\lim _{n} X_{n}$. Then the homology of $X_{n}$ is $R /\left(x_{0}, \ldots, x_{n-1}\right)$ concentrated in degree zero, and so $X_{\infty}$ is a projective resolution of $k=\lim _{n} R /\left(x_{0}, \ldots, x_{n-1}\right)$.

Since $S$ is finite dimensional, $\operatorname{Ext}_{R}^{t}\left(D R^{J}, S\right)=D \operatorname{Tor}_{t}^{R}\left(D R^{J}, S\right)$, which is the dual of the degree $t$ homology of the chain complex $D R^{J} \otimes_{R} X_{\infty}=$ $\lim _{n} D R^{J} \otimes_{R} X_{n}$. Since $X_{n}$ is a complex of finitely generated $R$-modules,

$$
D R^{J} \otimes_{R} X_{\infty}=\underline{\lim _{\longrightarrow}}\left(\left(D R \otimes_{R} X_{n}\right)^{J}\right) .
$$

So to prove that $\operatorname{Ext}_{R}^{t}\left(D R^{J}, k\right)=0$, it suffices to prove that $D R \otimes_{R} X_{n}$ has no homology in degree $t$ for sufficiently large $n$.

We shall show that the homology of $D R \otimes_{R} X_{n}$ is $D\left(R /\left(x_{0}, \ldots, x_{n-1}\right)\right)$, concentrated in degree $n$.

This is true for $n=0$. There is a short exact sequence

$$
0 \longrightarrow R /\left(x_{0}, \ldots, x_{m-1}\right) \stackrel{x_{m}}{\longrightarrow} R /\left(x_{0}, \ldots, x_{m-1}\right) \longrightarrow R /\left(x_{0}, \ldots, x_{m}\right) \longrightarrow 0,
$$

so if it is true for $n=m$ then it is true for $n=m+1$.

There are also non-Noetherian commutative rings for which injectives do generate. For example, if $R=k\left[x_{0}, x_{1}, \ldots\right] /\left(x_{0}, x_{1}, \ldots\right)^{2}$ is the quotient of the ring considered above by quadratic polynomials, then the methods of Section 7 can easily be adapted to show that injectives generate for $R$.

\section{The Finitistic Dimension COnJeCture}

From now on we specialize to the case of a finite dimensional algebra $A$ over a field $k$. As at the end of the previous section, we use the notation $D V$ to denote the $k$-dual of a vector space $V$. Also we denote by $\bmod -A$ the full subcategory of Mod- $A$ consisting of finitely generated modules. 
One simplifying factor is that if $A$ is a finite dimensional algebra then every injective $A$-module is a direct summand of a direct sum of copies of $D A$, so $\langle\operatorname{Inj}-A\rangle$ is generated by the single object $D A$.

We recall the definition of the big and little finitistic dimensions of a finite dimensional algebra $A$. If $M$ is an $A$-module then $\operatorname{pd}(M)$ denotes the projective dimension of $M$.

Definition 4.1. Let $A$ be a finite dimensional algebra.

The big finitistic dimension of $A$ is

$$
\operatorname{FinDim}(A)=\sup \{\operatorname{pd}(M) \mid M \in \operatorname{Mod}-A \text { and } \operatorname{pd}(M)<\infty\} .
$$

The little finitistic dimension of $A$ is

$$
\text { findim }(A)=\sup \{\operatorname{pd}(M) \mid M \in \bmod -A \text { and } \operatorname{pd}(M)<\infty\} .
$$

The finitistic dimension conjecture (for finite dimensional algebras) is the conjecture that $\operatorname{findim}(A)<\infty$. This was publicized as a question by Bass Bas60 in 1960 and is still open. The corresponding question for the big finitistic dimension is also open, and we shall distinguish between the two questions as follows.

Definition 4.2. Let $A$ be a finite dimensional algebra.

We say that $A$ satisfies the big finitistic dimension conjecture if $\operatorname{FinDim}(A)<\infty$.

We say that $A$ satisfies the little finitistic dimension conjecture if findim $(A)<\infty$.

Clearly $\operatorname{FinDim}(A) \geq$ findim $(A)$ and so if $A$ satisfies the big finitistic dimension conjecture then it satisfies the little finitistic dimension conjecture.

Huisgen-Zimmermann [HZ95] gives a good survey of the finitistic dimension conjecture and its history.

Theorem 4.3. Let $A$ be a finite dimensional algebra over a field $k$. If injectives generate for $A$ then $A$ satisfies the big finitistic dimension conjecture (and hence also the little finitistic dimension conjecture).

Proof. Suppose that $A$ does not satisfy the big finitistic dimension conjecture. Then there is an infinite family $\left\{M_{i} \mid i \in I\right\}$ of nonzero $A$-modules with $\operatorname{pd}\left(M_{i}\right) \neq \operatorname{pd}\left(M_{j}\right)$ for $i \neq j$. Let $d_{i}=\operatorname{pd}\left(M_{i}\right)$.

Let $P_{i}$ be a minimal projective resolution of $M_{i}$. Then $P_{i}\left[-d_{i}\right]$, considered as a cochain complex, is zero except in degrees 0 to $d_{i}$, and has cohomology concentrated in degree $d_{i}$.

Both $\bigoplus_{i} P_{i}\left[-d_{i}\right]$ and $\prod_{i} P_{i}\left[-d_{i}\right]$ have homology isomorphic to $M_{i}$ in degree $d_{i}$, and the natural inclusion

$$
\iota: \bigoplus_{i} P_{i}\left[-d_{i}\right] \rightarrow \prod_{i} P_{i}\left[-d_{i}\right]
$$

is a quasi-isomorphism.

However, $\iota$ is not a homotopy equivalence, since if it were then applying any additive functor to $\iota$ would give a quasi-isomorphism. Note that $P_{i}\left[-d_{i}\right] \otimes_{A}(A / \operatorname{rad}(A))$ has nonzero cohomology $\operatorname{Tor}_{d_{i}}^{A}\left(M_{i}, A / \operatorname{rad}(A)\right)$ in degree zero. Since taking the tensor product with a finitely presented module 
preserves both products and coproducts, the map on degree zero cohomology induced by $\iota \otimes_{A}(A / \operatorname{rad}(A))$ is the natural map

$$
\bigoplus_{i} \operatorname{Tor}_{d_{i}}^{A}\left(M_{i}, A / \operatorname{rad}(A)\right) \rightarrow \prod_{i} \operatorname{Tor}_{d_{i}}^{A}\left(M_{i}, A / \operatorname{rad}(A)\right),
$$

which is not an isomorphism.

Thus, if $C$ is the mapping cone of $\iota$, then $C$ is a bounded below complex of projective $A$-modules that is acyclic but not contractible.

The functor $-\otimes_{A} D A$ is an equivalence from the category of projective $A$-modules to the category of injective $A$-modules. Applying this functor to $C$, we get a bounded below complex $C \otimes_{A} D A$ of injective $A$-modules that is not contractible (since $-\otimes_{A} D A$ is an equivalence), and so not acyclic, since a bounded below complex of injectives is acyclic if and only if it is contractible.

Since $C$ is acyclic, $\operatorname{Hom}_{\mathcal{K}(A)}(A, C[t])=0$, and so

$$
\operatorname{Hom}_{\mathcal{K}(A)}\left(D A, C \otimes_{A} D A[t]\right)=0,
$$

for all $t \in \mathbb{Z}$.

Since $C \otimes_{A} D A$ is a bounded below complex of injectives,

$$
\operatorname{Hom}_{\mathcal{D}(A)}\left(D A, C \otimes_{A} D A[t]\right) \cong \operatorname{Hom}_{\mathcal{K}(A)}\left(D A, C \otimes_{A} D A[t]\right)=0 .
$$

But the objects $X$ of $\mathcal{D}(A)$ such that $\operatorname{Hom}_{\mathcal{D}(A)}\left(X, C \otimes_{A} D A[t]\right)=0$ for all $t$ form a localizing subcategory of $\mathcal{D}(A)$, and therefore $C \otimes_{A} D A$ is not in $\langle\operatorname{Inj}-A\rangle$. So injectives do not generate for $A$.

The proof of the last theorem also provides an equivalent form of the big finitistic dimension conjecture that is similar to an equivalent form of "injectives generate" that follows by a standard application of Bousfield localization.

We define the right perpendicular category $D A^{\perp}$ of $D A$ to be the full subcategory of $\mathcal{D}(A)$ consisting of the objects $X$ such that, for all $t \in \mathbb{Z}$, $\operatorname{Hom}_{\mathcal{D}(A)}(D A, X[t])=0$. As usual, $\mathcal{D}^{+}(A)$ denotes the full triangulated subcategory of $\mathcal{D}(A)$ consisting of complexes with cohomology bounded below in degree.

Theorem 4.4. Let $A$ be a finite dimensional algebra over a field $k$.

(a) Injectives generate for $A$ if and only if $D A^{\perp}=\{0\}$.

(b) A satisfies the big finitistic dimension conjecture if and only if

$$
D A^{\perp} \cap \mathcal{D}^{+}(A)=\{0\} .
$$

Proof. If $X$ is an object of $D A^{\perp}$, then the class ${ }^{\perp} X$ of objects $Y$ for which $\operatorname{Hom}(Y, X[t])=0$ for all $t \in \mathbb{Z}$ is a localizing subcategory of $\mathcal{D}(A)$ containing $D A$, so if injectives generate for $A$ then $X$ is in ${ }^{\perp} X$, and so the existence of the identity map for $X$ shows that $X \cong 0$. The converse of part (a) is just "Bousfield localization" Bou79]. For any object $X$ of $\mathcal{D}(A)$, there is a distinguished triangle

$$
\Gamma X \rightarrow X \rightarrow L X \rightarrow \Gamma X[1]
$$

such that $\Gamma X$ is in $\langle\operatorname{Inj}-A\rangle$ and $L X$ is in $D A^{\perp}$. If $D A^{\perp}=\{0\}$ then for every object $X$ of $\mathcal{D}(A), X \cong \Gamma X \in\langle\operatorname{Inj}-A\rangle$ and so injectives generate for $A$. 
That Bousfield localization applies in this situation (or more generally for a localizing subcategory generated by a single object in a compactly generated triangulated category) seems to be well-known, an explicit proof can be found in [ATJLSS00, Proposition 5.7] or, for a more general statement, in [Kra10, Theorem 7.2.1].

For (b), the proof of Theorem 4.3 shows that if $A$ does not satisfy the big finitistic dimension conjecture then there is a nonzero object of $\mathcal{D}^{+}(A)$ in $D A^{\perp}$.

The argument for the converse emerged from a conversation with Henning Krause. Suppose $X:=X^{*}$ is a nonzero object of $D A^{\perp} \cap \mathcal{D}^{+}(A)$. Without loss of generality, $X$ is a complex of injectives concentrated in positive degrees and $X^{0} \rightarrow X^{1}$ is not a split monomorphism. Since $X$ is a bounded below complex of injectives,

$$
\operatorname{Hom}_{\mathcal{K}(A)}(D A, X[t]) \cong \operatorname{Hom}_{\mathcal{D}(A)}(D A, X[t])=0
$$

for all $t \in \mathbb{Z}$.

Applying the equivalence $\operatorname{Hom}_{A}(D A,-)$ between injective and projective $A$-modules to $X$ we get a complex $Y^{*}:=\operatorname{Hom}_{A}(D A, X)$ of projective $A$-modules concentrated in non-negative degrees such that, for all $t \in \mathbb{Z}$, $\operatorname{Hom}_{\mathcal{K}(A)}\left(A, Y^{*}[t]\right)=0$ (i.e., $Y^{*}$ is acyclic) and such that $Y^{0} \rightarrow Y^{1}$ is not a split monomorphism.

Hence, for any $t>0$

$$
\sigma^{\leq t} Y:=\cdots \rightarrow 0 \rightarrow Y^{0} \rightarrow Y^{1} \rightarrow \cdots \rightarrow Y^{t} \rightarrow 0 \rightarrow \ldots
$$

is the projective resolution of an $A$-module with projective dimension $t$, and so $A$ does not satisfy the big finitistic dimension conjecture.

\section{The COlOCALIZING SUbCATEgORY Generated By PROJECTIVES}

As well as considering whether $\mathcal{D}(R)$ is generated as a triangulated category with arbitrary small coproducts by the injective modules, it makes sense to ask the dual question: is $\mathcal{D}(R)$ generated as a triangulated category with arbitrary small products by the projective modules.

For general rings there seems to be no direct connection between the two questions, but for finite dimensional algebras there is one implication.

Proposition 5.1. Let $A$ be a finite dimensional algebra over a field for which $\mathcal{D}(A)$ is generated by the injective modules as a triangulated category with arbitrary coproducts. Then $\mathcal{D}\left(A^{o p}\right)$ is generated by the projective modules as a triangulated category with arbitrary products.

Proof. Consider the full subcategory $\mathcal{X}$ of $\mathcal{D}(A)$ consisting of the objects $X$ for which the dual $D X$ is in the colocalizing subcategory of $\mathcal{D}\left(A^{o p}\right)$ generated by projectives. Then $\mathcal{X}$ is a localizing subcategory of $\mathcal{D}(A)$ that contains $D A$, and so $\mathcal{X}=\mathcal{D}(A)$. Hence $D A$ is in the colocalizing subcategory of $\mathcal{D}\left(A^{o p}\right)$ generated by projectives, and $D A$ generates $\mathcal{D}\left(A^{o p}\right)$ as a triangulated category with arbitrary products.

In fact, the proof of Theorem 4.3 can be adapted to show that this potentially weaker condition is sufficient to prove the finitistic dimension conjecture. 
Proposition 5.2. Let $A$ be a finite dimensional algebra over a field. If projectives generate $\mathcal{D}\left(A^{o p}\right)$ as a triangulated category with arbitrary products, then A satisfies the big finitistic dimension conjecture.

We'll only sketch the proof, which is very similar to that of Theorem 4.3 .

Proof. If there is an infinite family $\left\{M_{i} \mid i \in I\right\}$ of nonzero $A$-modules with $\operatorname{pd}\left(M_{i}\right)=d_{i} \neq d_{j}=\operatorname{pd}\left(M_{j}\right)$ for $i \neq j$ then $\left\{D M_{i} \mid i \in I\right\}$ is a family of nonzero $A^{o p}$-modules with $\operatorname{id}\left(D M_{i}\right)=d_{i}$, so $\operatorname{id}\left(D M_{i}\right) \neq \operatorname{id}\left(D M_{j}\right)$ for $i \neq j$.

Let $I_{i}$ be a minimal injective resolution of $D M_{i}$. Then the mapping cone $C$ of the natural inclusion

$$
\bigoplus_{i} I_{i}\left[d_{i}\right] \rightarrow \prod_{i} I_{i}\left[d_{i}\right]
$$

is a bounded above acyclic but not contractible complex of injective $A^{o p_{-}}$ modules. Since $C$ is acyclic, $\operatorname{Hom}_{\mathcal{K}\left(A^{o p}\right)}(C, D A[t])=0$, and so (applying the equivalence between injective and projective modules)

$$
\operatorname{Hom}_{\mathcal{K}\left(A^{o p}\right)}\left(\operatorname{Hom}_{A^{o p}}(D A, C), A[t]\right)=0
$$

for all $t \in \mathbb{Z}$.

Since $\operatorname{Hom}_{A^{o p}}(D A, C)$ is a bounded above complex of projective $A^{o p_{-}}$ modules,

$\operatorname{Hom}_{\mathcal{D}\left(A^{o p}\right)}\left(\operatorname{Hom}_{A^{o p}}(D A, C), A[t]\right)=\operatorname{Hom}_{\mathcal{K}\left(A^{o p}\right)}\left(\operatorname{Hom}_{A^{o p}}(D A, C), A[t]\right)=0$.

But the objects $X$ such that $\operatorname{Hom}_{\mathcal{D}\left(A^{o p}\right)}\left(\operatorname{Hom}_{A^{o p}}(D A, C), X[t]\right)=0$ for all $t \in \mathbb{Z}$ form a colocalizing subcategory of $\mathcal{D}\left(A^{o p}\right)$, and therefore the colocalizing subcategory generated by $A$ does not contain $\operatorname{Hom}_{A^{o p}}(D A, C)$.

The only real reason for focusing on generation by injectives in this paper, rather than the dual condition, is that localizing subcategories of $\mathcal{D}(A)$ and other triangulated categories are more familiar than colocalizing subcategories, and have received more attention.

\section{An EXAMPLE}

In this section we shall prove that injectives generate for a particular example of a finite dimensional algebra $A$ to illustrate some of the elementary methods that can be used.

Our strategy will be to explicitly build up a collection of objects of $\langle\operatorname{Inj}-A\rangle$ until we have shown that all the simple $A$-modules are in $\langle\operatorname{Inj}-A\rangle$.

Lemma 6.1. If $A$ is a finite dimensional algebra, and every simple $A$ module is in $\langle\mathrm{Inj}-A\rangle$, then injectives generate for $A$.

Proof. Every semisimple module is a coproduct of simple modules, and therefore in the localizing subcategory generated by simple modules. So, by induction on the radical length, every module is in the localizing subcategory generated by simple modules.

The algebra $A$ that we shall consider is borrowed from an example in a paper of Goodearl and Huisgen-Zimmermann GHZ98 that we shall discuss further in the next section. There is no particular motive behind choosing this algebra for our example, and in fact, owing to differences in left/right 
conventions, we actually consider the opposite algebra to the one that they studied.

$A$ is a binomial relation algebra: the quotient $A=k Q / I$ of the path algebra of a quiver $Q$ (with six vertices labelled $1, \ldots, 6$ ) by an admissible ideal $I$ generated by some paths and some elements of the form $p-\lambda q$, where $p$ and $q$ are paths and $\lambda$ is a nonzero scalar. For vertex $i$, the corresponding idempotent (trivial path) of $A$ will be denoted by $e_{i}$, the indecomposable projective $e_{i} A$ by $P_{i}$, the indecomposable injective $D\left(A e_{i}\right)$ by $I_{i}$, and the simple module $P_{i} / \operatorname{rad}\left(P_{i}\right) \cong \operatorname{soc}\left(I_{i}\right)$ by $S_{i}$.

We use diagrams to describe modules in a similar way to GHZ98. The indecomposable projective modules $P_{1}, \ldots, P_{6}$ are described by the following diagrams.
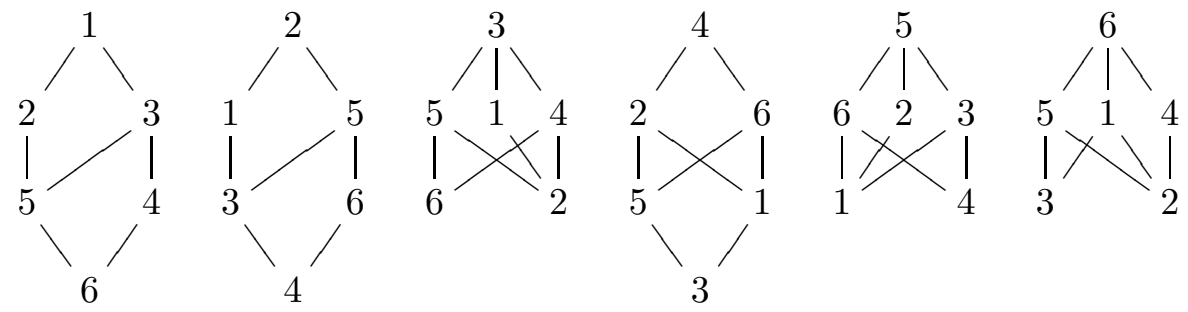

The numbers represent the elements of a basis for the module, each of which is annihilated by all but one of the idempotents $e_{i}$, that one being the one with the same number. Lines joining two numbers indicate that an arrow of $Q$ maps the basis element nearer the top of the diagram to a nonzero scalar multiple of the basis element nearer the bottom. For all pairs $(i, j)$ of vertices the quiver $Q$ has at most one arrow from vertex $i$ to vertex $j$, so there is never any ambiguity about which arrow is meant. The absence of a line indicates that a corresponding arrow acts as zero. So the top two layers of the diagrams for the indecomposable projectives tells us what the quiver is (e.g., there are two arrows starting at vertex 1, one of which ends at vertex 2 and one at vertex 3 ), and the rest of the diagram determines the relations apart from the precise values of the scalars $\lambda$, which we shall not need to know.

Not every module can be represented by such a diagram, but all the modules we need to consider can be, and in a way that determines the module up to isomorphism.

The projectives $P_{1}=I_{6}, P_{2}=I_{4}$ and $P_{4}=I_{3}$ are also injective, and the other indecomposable injectives $I_{1}, I_{2}$ and $I_{5}$ are described by the following diagrams.
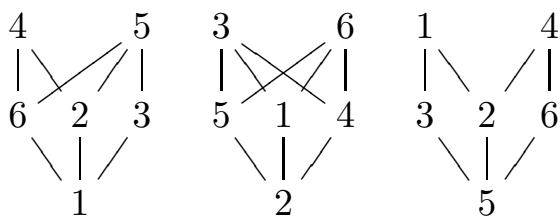
Let

$$
\left.M_{a}=\left.\right|_{6} ^{4} \text { and } \quad M_{b}=2 \backslash_{5}^{1}\right\rangle^{3}
$$

so that there are short exact sequences

$$
0 \rightarrow M_{a} \rightarrow I_{6} \rightarrow M_{b} \rightarrow 0
$$

and

$$
0 \rightarrow M_{b} \rightarrow I_{5} \rightarrow M_{a} \rightarrow 0 .
$$

Splicing copies of these short exact sequences, we get exact sequences

$$
\cdots \rightarrow I_{6} \rightarrow I_{5} \rightarrow I_{6} \rightarrow I_{5} \rightarrow M_{a} \rightarrow 0
$$

and

$$
\cdots \rightarrow I_{5} \rightarrow I_{6} \rightarrow I_{5} \rightarrow I_{6} \rightarrow M_{b} \rightarrow 0,
$$

so that $M_{a}$ and $M_{b}$ are quasi-isomorphic to bounded above complexes of injectives and are therefore in $\langle\operatorname{Inj}-A\rangle$ by Proposition 2.1(h).

Now let

$$
M_{c}=1>_{3}^{2} /^{5}
$$

so that there is a short exact sequence

$$
0 \rightarrow M_{c} \rightarrow I_{3} \rightarrow M_{a} \rightarrow 0 .
$$

Since the second and third terms are in $\langle\operatorname{Inj}-A\rangle, M_{c}$ is also in $\langle\operatorname{Inj}-A\rangle$ by Proposition 2.1(a).

Let

$$
M_{d}=\left.\right|_{4} ^{6}
$$

so that there is a short exact sequence

$$
0 \rightarrow M_{d} \rightarrow I_{4} \rightarrow M_{c} \rightarrow 0,
$$

proving that $M_{d}$ is in $\langle\operatorname{Inj}-A\rangle$.

There are exact sequences

$$
\cdots \rightarrow M_{d} \rightarrow M_{a} \rightarrow M_{d} \rightarrow M_{a} \rightarrow S_{4} \rightarrow 0
$$

and

$$
\cdots \rightarrow M_{a} \rightarrow M_{d} \rightarrow M_{a} \rightarrow M_{d} \rightarrow S_{6} \rightarrow 0,
$$

so $S_{4}$ and $S_{6}$ are also in $\langle\operatorname{Inj}-A\rangle$.

Let

$$
M_{e}=\backslash_{1}^{6} /^{3} \text { and } M_{f}=\backslash_{2}^{4} /^{5}
$$

There are short exact sequences

$$
0 \rightarrow M_{e} \rightarrow I_{1} \rightarrow M_{f} \rightarrow 0
$$


and

$$
0 \rightarrow M_{f} \rightarrow I_{2} \rightarrow M_{e} \rightarrow 0,
$$

so as above we can deduce that $M_{e}$ and $M_{f}$ are in $\langle\operatorname{Inj}-A\rangle$, and since we already know that $S_{4}$ and $S_{6}$ are in $\langle\operatorname{Inj}-A\rangle$ we can deduce that

$$
M_{g}=\left.\right|_{1} ^{3} \text { and } \quad M_{h}=\left.\right|_{2} ^{5}
$$

are in $\langle\operatorname{Inj}-A\rangle$.

Let

$$
M_{i}=\left.\right|_{5} ^{2} \text { and } \quad M_{j}=\begin{aligned}
& 1 \\
& 3 \\
& 4
\end{aligned}
$$

The short exact sequences

$$
0 \rightarrow M_{i} \rightarrow I_{6} \rightarrow M_{j} \rightarrow 0
$$

and

$$
0 \rightarrow M_{j} \rightarrow I_{4} \rightarrow M_{i} \rightarrow 0
$$

allow us to deduce that $M_{i}$ and $M_{j}$, and hence also

$$
M_{k}=\left.\right|_{5} ^{2} \quad \text { and } \quad M_{l}=\left.\right|_{3} ^{1}
$$

are in $\langle\operatorname{Inj}-A\rangle$. Finally $S_{1}$ is in $\langle\operatorname{Inj}-A\rangle$ because of the exact sequence

$$
\cdots \rightarrow M_{g} \rightarrow M_{l} \rightarrow M_{g} \rightarrow M_{l} \rightarrow S_{1} \rightarrow 0,
$$

and similarly the remaining simple modules $S_{2}, S_{3}$ and $S_{5}$ are in $\langle\operatorname{Inj}-A\rangle$.

\section{Some methods to Show that injectives Generate}

In this section we shall describe some methods to prove, for particular algebras and certain classes of algebras, that injectives generate. This includes classes that have long been known to satisfy the finitistic dimension conjecture, including some classes where the finitistic dimension conjecture is obvious, but the fact that injectives generate is less so. There are other classes of algebras which are known to satisfy the finitistic dimension conjecture (again, sometimes for obvious reasons), for which I do not know whether injectives generate.

As in Section 6 the strategy that we shall generally adopt is to show that all the simple modules are in the localizing subcategory generated by injectives, by giving explicit constructions.

We shall frequently be considering syzygies, and more particularly cosyzygies of modules, so we start with some notation. Throughout this section, $A$ will always be a finite dimensional algebra over a field.

Let $M$ be an $A$-module. For $i \geq 0$, the $i$ th syzygy $\Omega^{i} M$ of $M$ is the kernel of $d_{i-1}$ in a minimal projective resolution

$$
\ldots \stackrel{d_{3}}{\longrightarrow} P_{2} \stackrel{d_{2}}{\longrightarrow} P_{1} \stackrel{d_{1}}{\longrightarrow} P_{0} \stackrel{d_{0}}{\longrightarrow} M \stackrel{d_{-1}}{\longrightarrow} 0
$$


of $M$, and the $i$ th cosyzygy $\Sigma^{i} M$ is the kernel of $d^{i}$ in a minimal injective resolution

$$
0 \longrightarrow M \longrightarrow I^{0} \stackrel{d^{0}}{\longrightarrow} I^{1} \stackrel{d^{1}}{\longrightarrow} I^{2} \longrightarrow \ldots
$$

of $M$.

The importance of syzygies and cosyzygies to the finitistic dimension conjecture has long been recognized. We shall borrow some terminology and ideas from Goodearl and Huisgen-Zimmermann GHZ98, which itself builds on previous ideas of Jans Jan61, Colby and Fuller [CF90, Igusa and Zacharia [IZ90], Kirkman, Kuzmanovich and Small [KKS92, Wilson Wil83] and others. Goodearl and Huisgen-Zimmermann work primarily with syzygies, often for the opposite algebra, but for the connection with the localizing subcategory generated by injectives it is more convenient for us to use cosyzygies, so our definitions will be roughly dual to theirs. When considering syzygies and cosyzygies of finitely generated modules, which will usually be the case, they will be precisely dual.

We shall use the standard notation that $\operatorname{add}(X)$, for a module $X$, means the class of modules that are direct summands of finite direct sums of copies of $M$, and more generally, if $\mathcal{X}$ is a collection of modules, then $\operatorname{add}(\mathcal{X})$ is the class of modules that are direct summands of finite direct sums of copies of modules from $\mathcal{X}$.

Definition 7.1. Let $M$ be a finitely generated A-module. We say that $M$ has finite cosyzygy type if there is a finite set $\mathcal{X}$ of indecomposable $A$ modules such that, for every $t \geq 0, \Sigma^{t} M$ is in $\operatorname{add}(\mathcal{X})$.

Proposition 7.2. Suppose that a finitely generated module $M$ for a finite dimensional algebra $A$ has finite cosyzygy type. Then $M$ is in $\langle\operatorname{Inj}-A\rangle$.

Proof. For $n \geq 0$ let $\mathcal{X}_{n}$ be the class of indecomposable $A$-modules that occur as a direct summand of $\Sigma^{t} M$ for some $t \geq n$. Since $M$ has finite cosyzygy type, $\mathcal{X}_{0}$ contains only finitely many isomorphism classes. Also

$$
\cdots \subseteq \mathcal{X}_{n+1} \subseteq \mathcal{X}_{n} \subseteq \cdots \subseteq \mathcal{X}_{0},
$$

so there is some $n$ for which $\mathcal{X}_{m}=\mathcal{X}_{n}$ for all $m \geq n$.

Thus every module in $\operatorname{add}\left(\mathcal{X}_{n}\right)$ occurs as a direct summand of $\Sigma^{m} M$ for infinitely many $m \geq n$, and so if $X$ is the direct sum of countably many modules from each isomorphism class of indecomposable modules in $\operatorname{add}\left(\mathcal{X}_{n}\right)$, then

$$
\bigoplus_{m \geq n} \Sigma^{m} M \cong X \cong \bigoplus_{m>n} \Sigma^{m} M .
$$

Taking the coproduct of the short exact sequences

$$
0 \rightarrow \Sigma^{m} M \rightarrow I^{m} \rightarrow \Sigma^{m+1} M \rightarrow 0
$$

for $m \geq n$ gives a short exact sequence

$$
0 \rightarrow X \rightarrow I \rightarrow X \rightarrow 0
$$

where $I$ is injective.

Splicing together infinitely many copies of this short exact sequence gives an exact sequence

$$
\cdots \rightarrow I \rightarrow I \rightarrow I \rightarrow X \rightarrow 0 \rightarrow \ldots
$$


Hence $X$ is quasi-isomorphic to a bounded above complex of injectives, and so $X$ is in $\langle\operatorname{Inj}-A\rangle$ by Proposition 2.1(h).

By Proposition 2.1(e), $\Sigma^{n} M$ is in $\langle\operatorname{Inj}-A\rangle$, since it is a direct summand of $X$.

Since $M$ is quasi-isomorphic to the complex

$$
\cdots \rightarrow 0 \rightarrow I^{0} \rightarrow I^{1} \rightarrow \cdots \rightarrow I^{n-1} \rightarrow \Sigma^{n} M \rightarrow 0 \rightarrow \ldots,
$$

which is in $\langle\operatorname{Inj}-A\rangle$ by Proposition 2.1(g), $M$ is in $\langle\operatorname{Inj}-A\rangle$ by Proposition 2.1(c).

Corollary 7.3. If the simple A-modules have finite cosyzygy type, then injectives generate for $A$.

Proof. By Proposition 7.2 every simple $A$-module is in $\langle\operatorname{Inj}-A\rangle$, and so by Lemma 6.1, injectives generate for $A$.

There are some classes of algebras for which this condition is obviously satisfied.

Corollary 7.4. Injectives generate for any finite dimensional algebra $A$ with finite representation type.

Proof. Clearly Corollary 7.3 applies.

Corollary 7.5. Injectives generate for any finite dimensional algebra $A$ with $\operatorname{rad}^{2}(A)=0$.

Proof. If $S$ is a simple $A$-module, then $\Sigma^{i} S$ is semisimple for every $i$, and so Corollary 7.3 applies.

Recall that a monomial algebra is the path algebra of a quiver modulo an admissible ideal generated by paths.

Corollary 7.6. Injectives generate for a monomial algebra $A$.

Proof. The opposite algebra of a monomial algebra is also a monomial algebra, and the simple modules for a monomial algebra are syzygy finite [IZ90, HZ92]. So Corollary 7.3 applies.

This implies the fact, which was first proved by Green, Kirkman and Kuzmanovich [GKK91, that every monomial algebra satisfies the finitistic dimension conjecture.

Here is another definition that is simply the dual of a definition of Goodearl and Huisgen-Zimmermann [GHZ98].

Definition 7.7. Let $M$ be a finitely generated A-module. If there is some $n \geq 0$ such that every non-injective indecomposable direct summand of $\Sigma^{n} M$ occurs as a direct summand of $\Sigma^{t} M$ for infinitely many $t$, then the cosyzygy repetition index of $M$ is the least such $n$. If there is no such $n$ then the cosyzygy repetition index of $M$ is $\infty$.

Clearly a module with finite cosyzygy type also has finite cosyzygy repetition index, since we could take the $n$ in the definition of cosyzygy repetition index to be the $n$ that occurs in the proof of Proposition 7.2.

Proposition 7.8. If a finitely generated module $M$ for a finite dimensional algebra $A$ has finite cosyzygy repetition index, then $M$ is in $\langle\operatorname{Inj}-A\rangle$. 
Proof. We shall suppose the cosyzygy repetition index of $M$ is $n$.

The proof is almost identical to that of Proposition 7.2, except that we'll take the coproduct of the short exact sequences

$$
0 \rightarrow \Sigma^{m} M \rightarrow I^{m} \rightarrow \Sigma^{m+1} M \rightarrow 0
$$

for $m$ strictly greater than $n$ in order to get a short exact sequence

$$
0 \rightarrow X \rightarrow I \rightarrow X \rightarrow 0 \text {. }
$$

One useful fact that Goodearl and Huisgen-Zimmermann GHZ98] prove is that the big finitistic dimension conjecture is true for $A$ if every simple module for the opposite algebra is a submodule of a module with finite syzygy type (or more generally with finite syzygy repetition index). There seems to be no obvious reason that a similar condition should imply that injectives generate for $A$ since, for example, this condition is obviously satisfied for local algebras (for which the finitistic dimension is also obvious since there are no non-projective modules with finite projective dimension), but proving that injectives generate for local algebras seems no easier than for general finite dimensional algebras.

However, there is a way of extracting information from the localizing subcategory generated by injectives, short of proving that it is the whole derived category, in order to prove that an algebra satisfies the big finitistic dimension conjecture, and this does generalize results of [GHZ98].

Theorem 7.9. Let $A$ be a finite dimensional algebra over a field. Suppose that, for every simple $A$-module $S$, there is an object $J_{S}$ of $\langle\operatorname{Inj}-A\rangle$ with a map $\alpha_{S}: J_{S} \rightarrow S$ in $\mathcal{D}(A)$ that is non-zero on cohomology (for example, this would be the case if there were a module $J_{S}$ in $\langle\operatorname{Inj}-A\rangle$ with a copy of $S$ in its head). Then A satisfies the big finitistic dimension conjecture.

Proof. Suppose that $A$ does not satisfy the big finitistic dimension conjecture. Then by Theorem 4.4 there is a nonzero object $X$ of $\mathcal{D}^{+}(A)$ in $D A^{\perp}$. Replacing $X$ by an injective resolution and shifting in degree, we can assume that $X$ is a complex of injective modules concentrated in positive degrees, with nonzero cohomology in degree zero.

Let $S$ be a simple submodule of $H^{0}(X)$, so there is a map $\beta: S \rightarrow X$ that is nonzero on cohomology, and let $\alpha_{S}: J_{S} \rightarrow S$ be the map guaranteed by the hypotheses of the theorem. Then the composition $\beta \alpha_{S}: J_{S} \rightarrow X$ is nonzero on cohomology, and therefore is a nonzero map in $\mathcal{D}(A)$ from an object of $\langle$ Inj- $A\rangle$ to an object of $D A^{\perp}$, giving a contradiction.

\section{Summary AND OPEn QUeSTIONS}

I know of no examples of finite dimensional algebras for which it is known that injectives do not generate.

If it were proven that injectives generate for all finite dimensional algebras, then in light of Theorem 4.3 some longstanding open problems would be settled. And perhaps an example where injectives do not generate might give a clue that would help find a counterexample to some other conjectures. 
For convenience, the following theorem summarizes various classes of finite dimensional algebras for which the results of this paper prove that injectives do generate. The list is not complete (we omit some of the more technical conditions that follow from Section 7) or efficient (some of the classes properly contain others).

Theorem 8.1. Let $A$ be a finite dimensional algebra over a field. If $A$ is in any of the following classes of algebras, then injectives generate for $A$.

(a) Commutative.

(b) Gorenstein.

(c) Finite global dimension.

(d) Self-injective.

(e) Finite representation type.

(f) Radical square zero.

(g) Monomial.

(h) Algebras derived equivalent to any of the above.

Proof. Finite dimensional algebras are Noetherian, so (a) follows from Theorem 3.3. An algebra is Gorenstein if projective modules have finite injective dimension and injective modules have finite projective dimension (whether one of the conditions implies the other is an open question), so (b) follows from Theorem 3.2. Both (c) and (d) are special cases of (b). Corollaries 7.4, 7.5 and 7.6 give (e), (f) and (g). Theorem 3.4 implies (h).

There are other classes of finite dimensional algebras for which the finitistic dimension conjecture is known to hold, but for which I don't know whether injectives generate. For example, local algebras satisfy the finitistic dimension conjecture for obvious reasons (there are no non-projective modules with finite projective dimension), but it doesn't seem any easier to prove that injectives generate for local algebras than it is for general algebras.

The little finitistic dimension conjecture has been proved for algebras with radical cube zero by Green and Huisgen-Zimmermann GHZ91 and for algebras with representation dimension three by Igusa and Todorov [IT05]. However, the big finitistic dimension conjecture does not seem to be known for these algebras, so maybe we should expect that proving that injectives generate should be harder than for other classes of algebras where the big finitistic dimension conjecture is known to hold.

Even if it turns out to be hard to find an example of a finite dimensional algebra for which injectives don't generate, it might be easier to find a right Noetherian ring. By Theorem 3.3 such an example would need to be noncommutative.

It would also be interesting to clarify the connection, briefly touched on in Section 5, between injectives generating the derived category as a localizing subcategory and projectives generating as a colocalizing subcategory, both for finite dimensional algebras and for general rings.

\section{REFERENCES}

[ATJLSS00] Leovigildo Alonso Tarrío, Ana Jeremías López, and María José Souto Salorio, Localization in categories of complexes and unbounded resolutions, Canad. J. Math. 52 (2000), no. 2, 225-247. MR 1755776 
[Bas60] Hyman Bass, Finitistic dimension and a homological generalization of semiprimary rings, Trans. Amer. Math. Soc. 95 (1960), 466-488. MR 0157984

[BN93] Marcel Bökstedt and Amnon Neeman, Homotopy limits in triangulated categories, Compositio Math. 86 (1993), no. 2, 209-234. MR 1214458

[Bou79] A. K. Bousfield, The localization of spectra with respect to homology, Topology 18 (1979), no. 4, 257-281. MR 551009

[CF90] R. R. Colby and K. R. Fuller, A note on the Nakayama conjectures, Tsukuba J. Math. 14 (1990), no. 2, 343-352. MR 1085203

[GHZ91] Edward L. Green and Birge Huisgen-Zimmermann, Finitistic dimension of Artinian rings with vanishing radical cube, Math. Z. 206 (1991), no. 4, 505526. MR 1100835

[GHZ98] K. R. Goodearl and B. Huisgen-Zimmermann, Repetitive resolutions over classical orders and finite-dimensional algebras, Algebras and modules, II (Geiranger, 1996), CMS Conf. Proc., vol. 24, Amer. Math. Soc., Providence, RI, 1998, pp. 205-225. MR 1648628

[GKK91] Edward L. Green, Ellen Kirkman, and James Kuzmanovich, Finitistic dimensions of finite-dimensional monomial algebras, J. Algebra 136 (1991), no. 1, 37-50. MR 1085118

[Hap91] Dieter Happel, Homological conjectures in representation theory of finite dimensional algebras, Sherbrook Lecture Notes Series, Université de Sherbrooke, 1991.

[Hap93] $\quad$ Reduction techniques for homological conjectures, Tsukuba J. Math. 17 (1993), no. 1, 115-130. MR 1233117

[Hop87] Michael J. Hopkins, Global methods in homotopy theory, Homotopy theory (Durham, 1985), London Math. Soc. Lecture Note Ser., vol. 117, Cambridge Univ. Press, Cambridge, 1987, pp. 73-96. MR 932260

[HZ92] Birge Huisgen-Zimmermann, Homological domino effects and the first finitistic dimension conjecture, Invent. Math. 108 (1992), no. 2, 369-383. MR 1161097

[HZ95] - The finitistic dimension conjectures - a tale of 3.5 decades, Abelian groups and modules (Padova, 1994), Math. Appl., vol. 343, Kluwer Acad. Publ., Dordrecht, 1995, pp. 501-517. MR 1378224

[IT05] Kiyoshi Igusa and Gordana Todorov, On the finitistic global dimension conjecture for Artin algebras, Representations of algebras and related topics, Fields Inst. Commun., vol. 45, Amer. Math. Soc., Providence, RI, 2005, pp. 201-204. MR 2146250

[IZ90] Kiyoshi Igusa and Dan Zacharia, Syzygy pairs in a monomial algebra, Proc. Amer. Math. Soc. 108 (1990), no. 3, 601-604. MR 1031675

[Jan61] J. P. Jans, Some generalizations of finite projective dimension, Illinois J. Math. 5 (1961), 334-344. MR 0183748

[Kel01] Bernhard Keller, Unbounded derived categories and homological conjectures, Talk at summer school on "Homological conjectures for finite dimensional algebras", Nordfjordeid, 2001.

[KKS92] E. Kirkman, J. Kuzmanovich, and L. Small, Finitistic dimensions of Noetherian rings, J. Algebra 147 (1992), no. 2, 350-364. MR 1161298

[Kra10] Henning Krause, Localization theory for triangulated categories, Triangulated categories, London Math. Soc. Lecture Note Ser., vol. 375, Cambridge Univ. Press, Cambridge, 2010, pp. 161-235. MR 2681709

[Nee92] Amnon Neeman, The chromatic tower for $D(R)$, Topology 31 (1992), no. 3, 519-532, With an appendix by Marcel Bökstedt. MR 1174255

[Wil83] George V. Wilson, Ultimately closed projective resolutions and rationality of Poincaré-Betti series, Proc. Amer. Math. Soc. 88 (1983), no. 2, 221-223. MR 695246

School of Mathematics, University of Bristol, Bristol BS8 1TW, UK

E-mail address: j.rickard@bristol.ac.uk 